\title{
Model Dependence of Solvent Separated Sodium Chloride Ion Pairs in Water-DMSO Mixtures
}

\author{
A. Asthana, A.K. Chowdhury, A.K. Das, and B.L. Tembe \\ Department of Chemistry, I.I.T. Bombay, \\ Powai, Mumbai 400 076, India
}

\begin{abstract}
Constrained molecular dynamics simulations have been used to investigate ion pairing in water - DMSO mixtures. The potentials of mean force between the sodium - chloride ion pair are constructed by estimating the mean forces between the ion pair at various interionic separations and then integrating the mean forces. Two compositions of the solvent mixture with DMSO mole fractions of 0.21 and 0.35 are considered. Two model potentials for water and DMSO have been considered. One of the main observations is that the contact ion pair which is dominantly present in both the individual solvents is conspicuously absent in the mixture compositions studied. While solvent separated ion pairs dominate in all the mixture compositions, there is a presence of a second solvent separated ion pair in the water-DMSO mixture of composition with mole fraction of DMSO $=0.21$. The potentials of mean force are verified by dynamical trajectories of the ion pair. The dynamics of the solvation shells has also been investigated in detail.
\end{abstract}

\section{Introduction}

Ion pairs play a very important role in a variety of chemical and biochemical reactions. The solvation of ions and ion pairs influences the nature of reactions in solution media. ${ }^{1-5}$ The concept of two ion pair states, namely, the contact ion pair (CIP) and the solvent separated ion pair (SSIP), was proposed independently by Winstein ${ }^{6}$ and by Fuoss. ${ }^{7}$ The general scheme for any solvolysis reaction or any reaction that involves participation of solvent can be represented by

$$
\mathrm{CA} \leftrightarrow \mathrm{C}^{+} \mid \mathrm{A}^{-} \leftrightarrow \mathrm{C}^{+} \| \mathrm{A}^{-} \leftrightarrow \mathrm{C}^{+}+\mathrm{A}^{-}
$$

where the $\mathrm{C}^{+} \mid \mathrm{A}^{-}$represents the CIP and $\mathrm{C}^{+} \| \mathrm{A}^{-}$represents the SSIP. The two ion pair states are characterized by the existence of well-defined barriers in the ion-ion potential of mean force (PMF) derived through methods such as constrained MD simulations. These ion pairs also exist in mixed solvents and the transformation of the CIP into SSIP or vice versa involves the motion over an energy barrier between these two sates.

The first attempt to perform model calculations of solvations of alkali halides in DMSO was made by Goldenberg et al. ${ }^{8}$ Energy calculations were carried out on a cluster of an ion with 4, 5 and 6 molecules of solvent and on ion pairs with 14 DMSO molecules respectively. Ten point charges and nine polarizable bonds represented a DMSO molecule. It was found that calculated ion solvation energies were in quantitative agreement with experimental data. 
The first real MD simulation of liquid DMSO represented by 216 rigid molecules was performed by Rao and Singh. ${ }^{9}$ They determined relative differences in free energies of solvation between two different solutes, including $\mathrm{Na}^{+}, \mathrm{K}^{+}, \mathrm{Cl}^{-}$and $\mathrm{Br}^{-}$ions, in DMSO as well as in water and methanol. Their study showed that strong ion-dipole interaction, and a well-defined co-ordination sphere of five DMSO is formed around $\mathrm{Na}^{+}$ion in liquid DMSO. $\mathrm{Cl}^{-}$ion was found to be surrounded by about seven DMSO molecules and there is no well-defined co-ordination. A detailed investigation of the solvation phenomena of sodium and chloride ion pairs in liquid DMSO was undertaken by Madhusoodanan and Tembe. ${ }^{10}$ The potential mean force, the structure of solvation shells around the $\mathrm{Na}^{+}-\mathrm{Cl}^{-}$pair and the dynamics of association of $\mathrm{Na}^{+}$$\mathrm{Cl}^{-}, \mathrm{Na}^{+}-\mathrm{Na}^{+}$and $\mathrm{Cl}^{-}-\mathrm{Cl}^{-}$pairs were studied by using the method of constrained MD. ${ }^{11-13}$

Neutron diffraction and MD simulation based on the data, generated from the neutron diffraction experiments, were performed by Luzar et al to study the structure of water-DMSO binary mixture. ${ }^{14}$ The results showed the DMSO oxygen to be strongly hydrogen bonded, but the methyl groups were surrounded by loose hydrogen bonded cages of water molecules. No evidence for hydrophobic association of DMSO was observed. Chalaris and Samios ${ }^{15}$ studied the dynamics of water-DMSO mixtures using different effective potential model for both water and DMSO. They used the SPC, SPC/E and TIPS2 models for water and the P2 model for DMSO. The SPC/E gave the good results for water self- diffusion coefficient at low DMSO concentrations.

One of the recent MD simulation study of DMSO-water mixtures containing either an ionic or a neutral atomic solute was performed by Chowdhury and Chandra. ${ }^{16}$ The diffusion coefficients of both ionic and neutral solutes was found out using SPC/E potential model for water and 4-site P2 model of Luzar and Chandler for representing the DMSO molecules ${ }^{14}$.

In the next section, we outline the methodology used in the present study. This is followed by the results and discussions and conclusions.

\section{Methodology}

Simulation studies have been carried out on systems consisting of sodium and chloride ions in water-DMSO mixtures. Positions and velocities are calculated using the Verlet algorithm with a sufficiently small time step so that the constraints of the rigidity of the solvent molecules are satisfied with little difficulty during the simulations. ${ }^{17}$ The simulation study considers both the ions in one cubic simulation box along with solvent molecules. To calculate the potentials of mean force between the ions, we first calculate the average force between the ions using

$$
F(r)=F^{\text {direct }}(r)+\Delta F(r)
$$

Where $\Delta \mathrm{F}(\mathrm{r})$ is the contribution to the mean force from the solvent and $\mathrm{F}{ }^{\text {direct }}(\mathrm{r})$ is the bare ion-ion potential. The mean solvent force $\Delta \mathrm{F}(\mathrm{r})$ is obtained by ensemble averaging the instantaneous solvent force $\Delta \mathrm{F}(\mathrm{r}, \mathrm{t})$ between the ions.

$$
\begin{gathered}
\Delta \mathrm{F}(\mathrm{r}, \mathrm{t})=\mu\left[\mathbf{F}_{\mathrm{Na}}^{+}(\mathrm{r}, \mathrm{t}) / \mathrm{m}_{\mathrm{Na}^{+}}+-\mathbf{F}_{\mathrm{Cl}^{-}}(\mathrm{r}, \mathrm{t}) / \mathrm{m}_{\mathrm{Cl}^{-}}\right] \cdot \hat{\mathrm{r}} \\
\Delta \mathrm{F}(\mathrm{r})=<\Delta \mathrm{F}(\mathrm{r}, \mathrm{t})>
\end{gathered}
$$


$\Delta \mathrm{F}(\mathrm{r})$ is calculated at various fixed distances between the ions (in the range of 2.5 to $8 \stackrel{\circ}{A}$ ). In Eq. (2) $\hat{r}$ is the unit vector between the sodium and the chloride ions. The masses of the ions are denoted by $\mathrm{m}_{\mathrm{Na}}+$ and $\mathrm{m} \mathrm{Cl}_{\mathrm{Cl}^{-}}$and $\mu$ is their reduced mass. Typically 20 to 50 ps of equilibration is followed by production runs of 100 ps.

The potential of mean force is obtained by integrating the mean force as

$$
W(r)=-\int_{r_{0}}^{r} F(r) d r
$$

We need a suitable value of $\mathrm{W}(\mathrm{r})$ at $\mathrm{r}_{0}$ to complete the integration. This is done by assuming that at a distance of $r_{0}=8 \AA$,,$W(r)$ is given by the formula

$$
\mathrm{W}(\mathrm{r})=\mathrm{q}_{1} \mathrm{q}_{2} / \varepsilon \mathrm{r}
$$

Where $\varepsilon$ is the bulk dielectric constant. Its value is taken from the experimental data at the appropriate solvent mole fraction. The data is given in Table 1.

Our results for the SPC model of water and the P2 model of DMSO have been already reported. ${ }^{18-19}$ In the present article, we have used the SPC/E model of water and the P1 model of DMSO. While the parameters of the SPC/E model of water are rather close to those of the SPC model, the P1 model of DMSO differs considerably from the $\mathrm{P} 2$ model in that the charge on $\mathrm{S}$ in $\mathrm{P} 1$ is nearly four times the charge of the P2 model and the methyl groups in P1 are uncharged. The energy parameter of methyl in P1 is also twice that of the P2 model. There are two reasons for choosing the new parameters. One is that the new model has been to give better values of the individual ionic diffusion coefficients. The other reason is to find out the model dependence of the second SSIP proposed earlier.

The interparticle potentials are the usual Lennard Jones plus the Coulomb terms. The site-site ion-solvent and solvent -solvent potential is represented as

$$
\mathrm{U}_{\alpha \beta}(\mathrm{r})=\left(\mathrm{A}_{\alpha \beta} / \mathrm{r}^{12}\right)-\left(\mathrm{C}_{\alpha \beta} / \mathrm{r}^{6}\right)+\left(\mathrm{q}_{\alpha} \mathrm{q}_{\beta} / \mathrm{r}\right)
$$

Where $\alpha$ and $\beta$ denote a pair of interaction sites on different molecules, $r$ is the site separation, $\mathrm{q}_{\alpha}$ is a point charge located at site $\alpha$. The terms $\mathrm{A}_{\alpha \beta}$ and $\mathrm{C}_{\alpha \beta}$ are given by

$$
\begin{aligned}
& \mathrm{A}_{\alpha \beta}=4 \varepsilon_{\alpha \beta}\left(\sigma_{\alpha \beta}\right)^{12} \\
& \mathrm{C}_{\alpha \beta}=4 \varepsilon_{\alpha \beta}\left(\sigma_{\alpha \beta}\right)^{6}
\end{aligned}
$$

Here, $\varepsilon_{\alpha \beta}$ and $\sigma_{\alpha \beta}$ are the energy and the distance parameters in the Lennard-Jones potential. The cross interactions have been obtained by using the Lorentz-Berthelot rules

$$
\begin{gathered}
\sigma_{\alpha \beta}=\frac{1}{2}\left(\sigma_{\alpha \alpha+} \sigma_{\beta \beta}\right) \\
\varepsilon_{\alpha \beta}=\left(\varepsilon_{\alpha \alpha} \varepsilon_{\beta \beta}\right)^{1 / 2}
\end{gathered}
$$

These parameters of the potentials used have been given in Table 1 . Table 2 gives the details of the simulation cells. 
Table 1. Site parameters for models of water and DMSO

\begin{tabular}{llllllll}
\hline Solvent & Site & $\begin{array}{c}\boldsymbol{E} \\
(10 \mathrm{~J} / \mathrm{mol})\end{array}$ & $\mathrm{O} / \mathrm{A}$ & $\mathrm{q} / \mathrm{e}^{*}$ & $\begin{array}{c}\boldsymbol{E} \\
(10 \mathrm{~J} / \mathrm{mol})\end{array}$ & $\mathrm{\sigma} / \mathrm{A}$ & $\mathrm{q} / \mathrm{e}^{*}$ \\
\hline \multirow{3}{*}{ Water } & $\mathrm{O}$ & 0.6052 & 3.156 & -0.82 & 0.6052 & 3.166 & -0.8476 \\
& $\mathrm{H}$ & 0.0 & 0.0 & 0.41 & 0.0 & 0.0 & 0.4238 \\
& & $\mathrm{RS} / \mathrm{P} 2$ & $\mathrm{RS} / \mathrm{P} 2$ & $\mathrm{RS} / \mathrm{P} 2$ & $\mathrm{P} 1$ & $\mathrm{P} 1$ & $\mathrm{P} 1$ \\
DMSO & $\mathrm{S}$ & 0.84403 & 3.56 & 0.139 & 0.99741 & 3.4 & 0.54 \\
& $\mathrm{O}$ & 0.27586 & 2.94 & -0.459 & 0.29922 & 2.80 & -0.54 \\
& $\mathrm{CH}_{3}$ & 0.66852 & 3.60 & 1.60 & 1.23 & 3.80 & 0.0 \\
\hline
\end{tabular}

* e is the magnitude of the electronic charge.

Table 2. Details of the two mixture compositions showing compositions, number of solvent molecules, box lengths, densities and the dielectric constants ${ }^{18,19}$

\begin{tabular}{cccccccc}
\hline Composition & $\mathrm{x}_{1}$ & $\mathrm{x}_{2}$ & $\mathrm{~N}_{1}$ & $\mathrm{~N}_{2}$ & $\mathrm{~L}(\AA)$ & $\rho\left(k g L^{-1}\right)$ & $\epsilon$ \\
\hline 1 & 0.21 & 0.79 & 53 & 197 & 22.784 & 1.0725 & 74.85 \\
2 & 0.35 & 0.65 & 88 & 162 & 24.764 & 1.0927 & 69.97 \\
\hline
\end{tabular}

\section{Results and Discussion}

The mean forces between the ions for the two compositions are shown in Figs 1 and 2 for compositions 1 and 2 respectively.

The direct force is attractive and the solvent contribution is repulsive in both the compositions. The total contribution lies between -10 to 5 (kT/ $\AA$ )in the distance range of 3.5 to $8 \AA$. Both these forces are integrated from $r_{0}$ to $r$ to get the potential of mean force (PMF). The asymptotic form of the PMF of the continuum model of the solvent mixture at $r=r_{0}$ is used during the integration. The results for the two mixtures are shown in Figs 3 and 4 respectively.

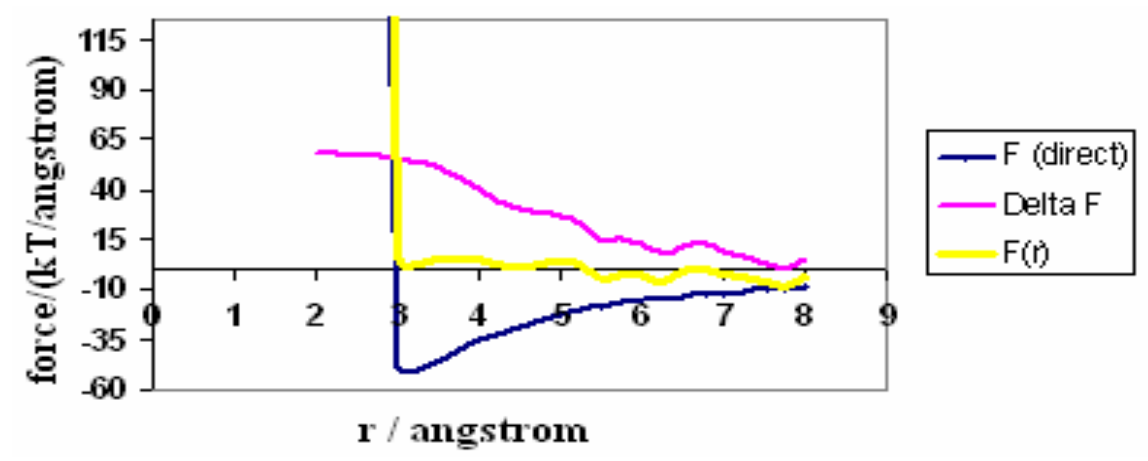

Fig. 1. The ion - ion force F (r), the contribution due to the solvent $F(r)$ and the total force between the ion pair for composition $1\left(\mathrm{x}_{\mathrm{DMSO}}=0.21\right)$ 


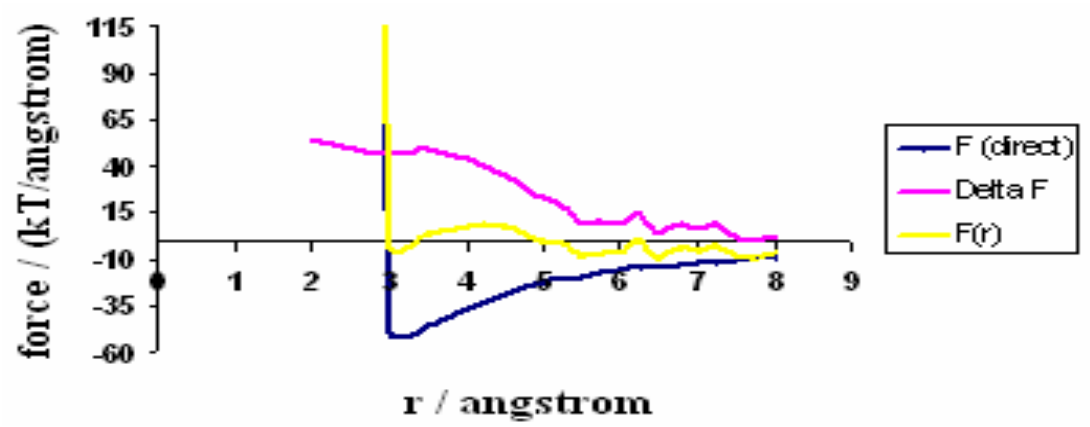

Fig. 2. The ion - ion force $F(r)$, the contribution due to the solvent $F(r)$ and the total force between the ion pair for composition $1\left(\mathrm{x}_{\text {DMSO }}=0.35\right)$

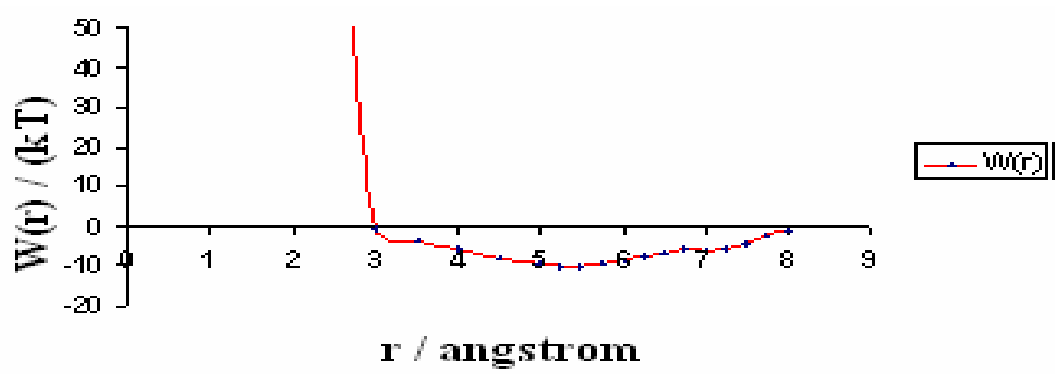

Fig. 3. Potential of mean force for the sodium chloride ion pair in composition $1\left(\mathrm{x}_{\text {DMSO }}=0.21\right)$

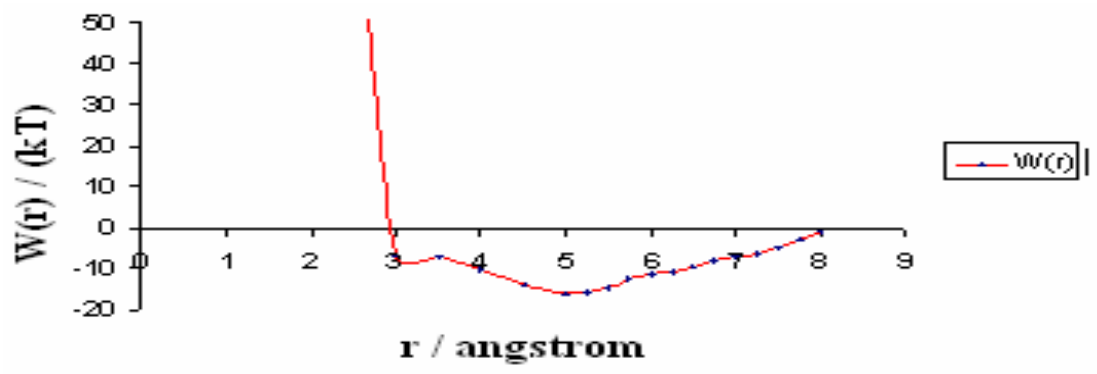

Fig. 4. Potential of mean force for the sodium chloride ion pair in composition $2(\mathrm{x}$ DMSO $=$ $0.35)$

Both the PMFs show the absence of a short range minimum around $3 \AA$ corresponding to the contact ion pair and the presence of a strong solvent-separated ion pair (SSIP) in the range of 4.5 to $5.5 \AA$ with a well depth of around $10 \mathrm{kT}$. The first composition (21\% DMSO) shows the presence of a second SSIP minimum near $7 \AA$, although this minimum is not separated from the first SSIP minimum with a very 
clear barrier. The verification of these PMFs was done by studying the ion pair trajectories which are initiated at various separations. Figs 5 and 6 show these ion pair trajectories for the mixtures.

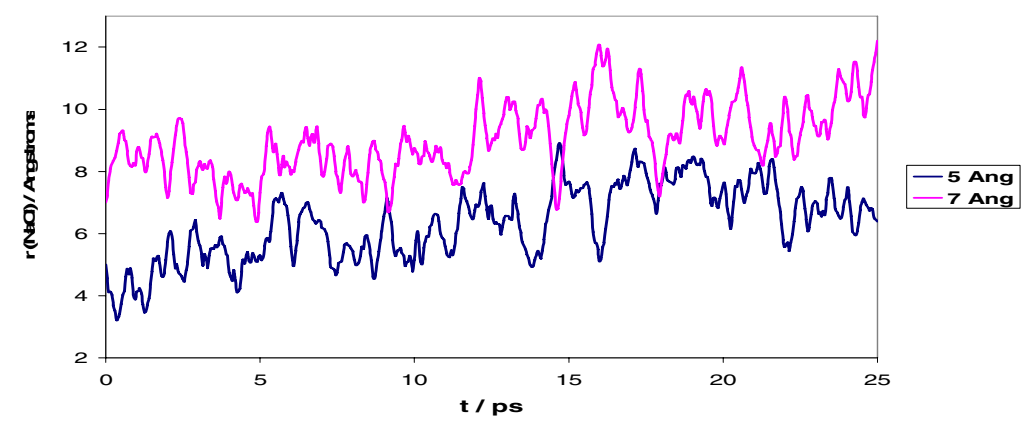

Fig. 5. Ion pair trajectories initiated at 5 and $7 \AA$ respectively for mixture $2\left(x_{\text {DMSO }}=0.21\right)$

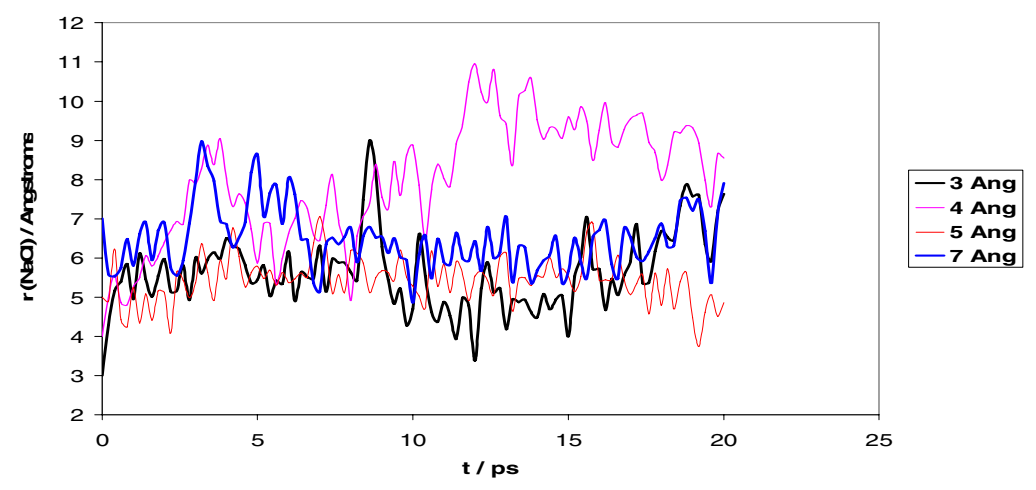

Fig. 6. Ion pair trajectories initiated at $3,4,5$ and $7 \AA$ for mixture $2\left(\mathrm{x}_{\text {DMSO }}=0.35\right)$

The trajectories are initiated with the ion pair separations of in the range of 3 to 7 $\AA$. The trajectories initiated at small ion pair separations of $3 \AA$ reach large ion separations ( of greater $4 \AA$ ) within a few ps. The ion pair trajectories initiated at large ion pair separations diffuse very slowly indicating that the long distance ion pairs have a very significant stability in the solvent mixtures. One of the reasons for this dominance of the solvent separated ion pair is the strong interaction between water and DMSO and the competition of both water and DMSO molecules to get into the first solvation shell of the ion pair. ${ }^{20}$ There appears to be a disruption of the contact ion pair formed in the pure solvents by the intrusion of the other solvent molecules in the first shell. The spread out solvent separated ion pair in the distance range of 4 to $6 \AA$ implies that there is a significant flux of molecules from the first shell to the bulk solvent and vice versa and this has been noticed in the simulations. The diffusivity of 
the trajectories in the present case $\mathrm{e}^{21}$ is similar to our previous work ${ }^{18,19}$ and we are investigating these aspects in greater detail.

\section{Conclusions}

The present results for the PMFs have a close similarity to the findings of the earlier model calculations. ${ }^{18}$ There is a striking absence of contact ions pairs and a dominance of solvent separated ions pairs. These are confirmed by detailed dynamical trajectories. While the parameters of the SPC/E model of water are rather close to those of the SPC model, the P1 model of DMSO differs considerably from the P2 model with respect to the charge on $\mathrm{S}$ and the size of the methyl groups. We are investigating the details of this clustering as well as the long time trajectories of the ion pairs. We may find larger deviations in the PMFs between the two models for compositions wherein the mole fractions of DMSO are larger. These studies are under way.

\section{References}

1. Hubbard, J., Wolynes, P.: The Chemical Physics of Solvation, Part C: (Ed) Dogonadze, R.R., Kalman, E.,Kornyshev, A. A., Ulstrup, J., Elsevier, New York (1988)

2. Buchner, R.:Dielectric Spectroscopy of Solutions, in: (eds) Samios, J,. Durov, V.A. Novel Approaches to the Structure and Dynamics of Liquids: Experiments, Theories and Simulations, NATO Science Ser. II: Mathematics, Physics and Chemistry, Vol. 133, pp. 265-288, Kluwer, Dordrecht (2004)

3. Bester-Rogac, M., Neueder, R., Barthel, J.: J. Solution Chem. 28 (1999) 1077-1092

4. Tongraar, A., Rode, B. M.: Phys.Chem.ChemPhys., 6 (2004) 411-416

5. Schwenk, C. F., Hofer, T. S., Rode, B. M.: J. Phys. Chem.A 108 (2004) 1509-1514

6. Winstein, S., Clippinger, E., Fainber, A. H., Robinson, G.C.: J. Am. Chem. Soc. 26 (1954) 1696.

7. Sadek, H., Fuouss, R.: J. Am. Chem. Soc., 76 (1954) 5897

8. Goldenberg, M.S., Kruus, P., Luk, S. F. K.: Can. J. Chem. 53 ( 1975) 1007

9. Rao, B. G., Singh, U. C.: J. Am. Chem.Soc. 112 (1990) 3803

10. Madhusoodanan, M.; Tembe, B.L.: J. Phys.Chem. 98 (1994) 7090

11. Guardia, E., Rey, R., Padro, J. A.: Chem. Phys. 155 (1991) 187

12. Sesse, G.;Guardia, E., Padro, J. A.: J. Chem. Phys. 99 (1995) 12647

13. Das, A. K., Madhusoodanan, M., Tembe, B. L.: J. Phys. Chem. A101 (1997), 2862

14. Luzar, A., Chandler, D.: J. Chem. Phys 98 (1993) 8160

15. Chalaris, M., Samois, J.: J. Mol. Liq., 98 (2002) 399

16. Chowdhury, S.; Chandra, A.: J. Chem. Phys. 119 (2003) 4360

17. Allen, M. P., Tildesley D. J.: Computer Simulation of Liquids, Oxford University Press (1987)

18. Das, A. K., Tembe, B. L.: J. Chem. Phys. 111 (1999) 7526-7536

19. Das, A. K.: Simulation Studies on the Sodium Chloride Ion Pair in DMSO and WaterDMSO Mixtures, Ph.D Thesis, IIT Bombay ( 1998)

20. Das, U.: "Molecular dynamics study of the solvation of ions in water-DMSO Mixtures", M.Sc. Thesis, Department of Chemistry, IIT Bombay, 2003

21. Asthana, A.: "Dynamics of ions and molecules in solvent mixtures like Water-DMSO", M.Sc. Thesis, Department of Chemistry, IIT Bombay, 2005 